\title{
Application of the continuous wavelet transform in periodic error
}

\section{compensation}

\author{
Chao Lu ${ }^{\text {a }}$, John R. Troutman ${ }^{\text {b }}$, Tony L. Schmitz ${ }^{\text {, }}$, Jonathan D. Ellis ${ }^{\text {c }}$, Joshua A. Tarbutton ${ }^{\text {a,* }}$ \\ ${ }^{a}$ Department of Mechanical Engineering, University of South Carolina, 300 Main Street, Columbia, SC 29208, USA \\ ${ }^{\mathrm{b}}$ Department of Mechanical Engineering and Engineering Science, University of North Carolina at Charlotte, Charlotte, NC 28223, USA \\ ${ }^{\mathrm{c}}$ Department of Mechanical Engineering and The Institute of Optics, University of Rochester, 235 Hopeman Building, Rochester, NY \\ 14627, USA
}

\begin{abstract}
This paper introduces a new discrete time continuous wavelet transform (DTCWT)-based algorithm, which can be implemented in real time to quantify and compensate periodic error for constant and non-constant velocity motion in heterodyne displacement measuring interferometry. It identifies the periodic error by measuring the phase and amplitude information at different orders (the periodic error is modeled as a summation of pure sine signals), reconstructs the periodic error by combining the magnitudes for all orders, and compensates the periodic error by subtracting the reconstructed error from the displacement signal measured by the interferometer. The algorithm is validated by comparing the compensated results with a traditional frequency domain approach for constant velocity motion. The algorithm demonstrates successful reduction of the first order periodic error amplitude from $4 \mathrm{~nm}$ to $0.24 \mathrm{~nm}$ (a 94\% decrease) and a reduction of the second order
\end{abstract}

\footnotetext{
* Corresponding author. Tel.: +1 803777 8236; fax: +1 803777 0106. E-mail address: JAT@ @sc.edu (J.A. Tarbutton).
} 
periodic error from $2.5 \mathrm{~nm}$ to $0.3 \mathrm{~nm}$ (an $88 \%$ decrease). The algorithm also reduces periodic errors for non-constant velocity motion overcoming limitations of existing methods.

\section{Keywords:}

Interferometry

Heterodyne

Periodic error

Signal processing

Wavelet transform

\section{Introduction}

Displacement measuring interferometry provides high resolution and accuracy for dimensional metrology and is used in a number of applications including semiconductor fabrication and linear stage calibration. Heterodyne (two-frequency) Michelson-type are often selected. The interference between the reference and measurement signals is observed at a photodetector, where current is generated proportional to the optical interference signal. The current is processed and converted to voltage and the phase between the reference 
signal and the signal after displacement is determined by phase-measuring electronics. The measured phase change is nominally linearly proportional to the displacement of the measurement target. However, errors and defects in the optical system components cause frequency mixing between the polarized reference and measurement arms. This frequency mixing causes periodic error to be superimposed on the measured displacement signal. For heterodyne interferometers, both first and second order periodic errors can occur, which correspond to one and two periods per displacement fringe. The periodic error can limit the accuracy of the heterodyne interferometer to the nanometer level (or higher) depending on the optical setup.

Previous research has demonstrated a frequency domain approach to periodic error identification [1-3], where the periodic error is measured by calculating the Fourier transform of the time domain data collected during constant velocity target motion. The periodic errors are then determined from the relative amplitudes of the peaks in the frequency spectrum. For an accelerating or decelerating motion, however, the Doppler frequency varies with velocity. In this case, the frequency domain approach is not wellsuited because the Fourier transform assumes stationary signals. To overcome this limitation, a wavelet-based analysis is applied here to measure and compensate periodic error.

A continuous wavelet transform (CWT) of a time domain signal provides information in both the temporal and frequency domains [4]. For example, calculating the Morlet CWT enables the frequency content of a signal to be observed at different times [5-7]. The CWT 
can be more informative than the Fourier transform because the CWT shows the relationship between frequency content and signal based on the wavelet scale and the time period. This enables the frequency and time information of the signal to be determined simultaneously by applying an appropriate wavelet. When applied to non-stationary signals, the CWT can supply accurate frequency information continuously.

In this work, a wavelet-based approach is used to capture both the temporal and spectral components of periodic error to allow for non-constant velocity error compensation. This paper discusses periodic error, the CWT in its general form, and introduces a new waveletbased approach to measure and compensate periodic error for both constant and nonconstant velocity target motions. The effectiveness of the wavelet-based approach to detecting and compensating periodic error is compared to the traditional Fourier-based approach for constant velocity motion. The effectiveness of the wavelet-based approach is also tested for non-constant velocity motion to demonstrate that utilizing wavelets allows for periodic error compensation for time-varying and frequency-varying errors.

\section{Background}

\subsection{Periodic error}

Heterodyne Michelson interferometers (Fig. 1) use a two-frequency laser source and separate the two optical frequencies into one fixed length and one variable length path via polarization. Ideally these two beams are linearly polarized and orthogonal so that only one 
frequency is directed toward each path. An interference signal is obtained by recombining the light from the two paths; this results in a measurement signal at the heterodyne (split) frequency of the laser source. This measurement signal is compared to the optical reference signal. Motion in the measurement arm causes a Doppler shift of the heterodyne frequency which is measured as a continuous phase shift that is proportional to displacement. In practice, due to misalignment of optical components, component imperfections, and elliptical polarization, undesirable frequency mixing occurs which yields periodic errors.

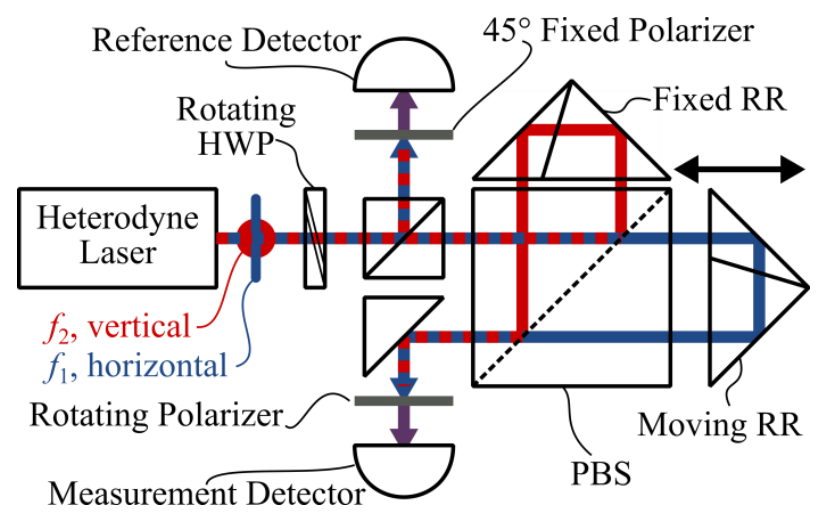

Fig. 1. Schematic of heterodyne interferometer setup. Optical components include: retroreflectors (RR), polarizing beam splitter (PBS), polarizers, half wave plate (HWP), and photodetectors.

Fedotova [8], Quenelle [9], and Sutton [10] first investigated periodic error in heterodyne Michelson interferometers. Subsequent studies of periodic error and its reduction have been reported in the literature [8-38]. Researchers have analyzed and applied different methods to measure and compensate periodic error, including the frequency domain approach [1-3] and several time domain measure-and-compensate algorithms [39-43]. 


\subsection{Continuous wavelet transform}

A wavelet function of time, $\psi(t)$, is a finite energy function with an average of zero and is usually normalized to a unit value [44],

$$
\begin{gathered}
\bar{\psi}(t)=\int_{-\infty}^{+\infty} \psi(t) d t=0, \\
\|\psi(t)\|^{2}=\int_{-\infty}^{+\infty}|\psi(t)| d t=1 .
\end{gathered}
$$

A wavelet family can be generated from a "mother" wavelet by translating it via the shift parmeter, $u \in \mathfrak{R}$ and dilating the wavelet via the scale parameter, $s>0$ (Fig. 2). This series of wavelets can be expressed as

$$
\psi_{u, s}(t)=\frac{1}{\sqrt{s}} \psi\left(\frac{t-u}{s}\right)
$$
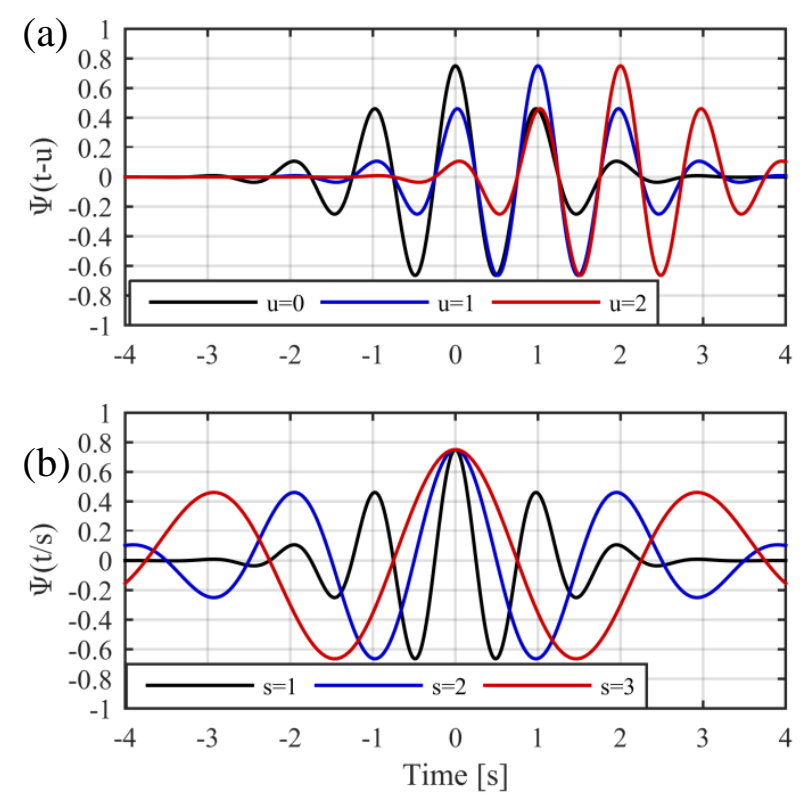
Fig. 2. Translation and dilation of the mother wavelet. (a) Shifting a wavelet in time is translation. (b) Stretching or squeezing a wavelet is dilation.

In this research, a continuous wavelet transform (CWT) is used to analyze the displacement signal, $x(t)$, which is measured by the heterodyne interferometer, with a wavelet function $\psi(t)$. For this one-dimensional signal, $x(t)$, the CWT result $W$ is defined as the convolution of $x(t)$ with a scaled and translated variation of the mother wavelet $\psi(t)$ via

$$
W x(u, s)=\int_{-\infty}^{+\infty} x(t) \psi_{u, s}^{*}(t) d t=\int_{-\infty}^{+\infty} x(t) \frac{1}{\sqrt{s}} \psi^{*}\left(\frac{t-u}{s}\right) d t
$$

where * indicates the complex conjugate. One property of CWT is its linearity,

$$
\left(W \sum_{i=1}^{N} \alpha_{i} x_{i}\right)(u, s)=\sum_{i=1}^{N} \alpha_{i}\left[W x_{i}(u, s)\right]
$$

which can be used to analyze a multi-component signal $x=\sum_{i=1}^{N} \alpha_{i} x_{i}$, where $\alpha_{i}(i=1 \ldots N)$

are constants. This property will be used in the algorithm to obtain periodic error amplitudes.

There are many types of wavelet functions available depending on the detection approach. Commonly used wavelets are the Haar, Daubechies, Meyer, Mexican Hat, and Morlet. Wavelets are selected based on the signal characteristic that can be extracted by the particular wavelet. For phase evaluation (i.e., for a sinusoidal model of periodic error, amplitude and phase are to be determined), the complex Morlet wavelet is suitable because it enables localization in both the time and frequency domains [45]. The frequency of the 
periodic error signal is located at the scale with the maximum wavelet coefficient and the phase information can be extracted based on the real and imaginary parts of this coefficient.

The complex Morlet wavelet is composed of a complex exponential multiplied by a Gaussian window (Fig. 3),

$$
\psi^{*}\left(\frac{t-u}{s}\right)=\pi^{-\frac{1}{4}} e^{i 2 \pi f_{0} \frac{t-u}{s}} e^{-\frac{1}{2}\left(\frac{t-u}{s}\right)^{2}},
$$

where $f_{0}$ is the central frequency of the mother wavelet.

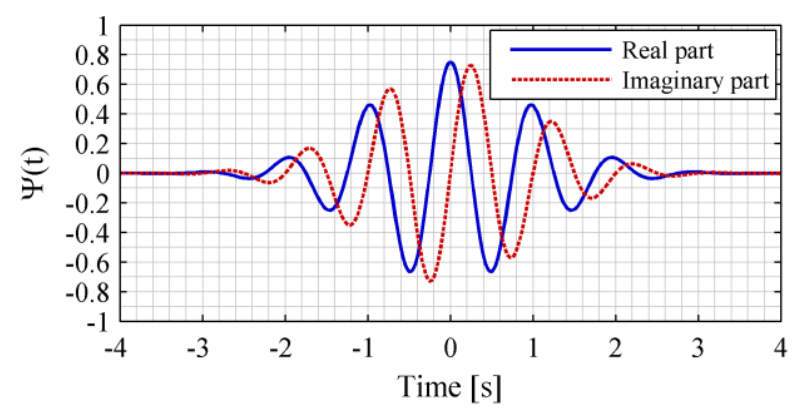

Fig. 3. The mother wavelet of the complex Morlet wavelet.

\section{Algorithm}

This section introduces the novel wavelet-based periodic error compensation algorithm in real-time, including all the calculation steps from receiving a new data point to outputting a compensated data point. In this research, only the periodic error is considered. For a motion of a moving retroreflector in a single pass interferometer, for example, Fig. 4 displays a simulated displacement of this motion from $25 \mu \mathrm{m}$ to $30 \mu \mathrm{m}$ with a velocity of $50 \mathrm{~mm} / \mathrm{min}$ 
in $0.006 \mathrm{~s}$, and superimposed periodic error with a first order magnitude of $4 \mathrm{~nm}$ and second order magnitude of $2.5 \mathrm{~nm}$. The nominal constant velocity motion is extracted to reveal only the remaining periodic error component.
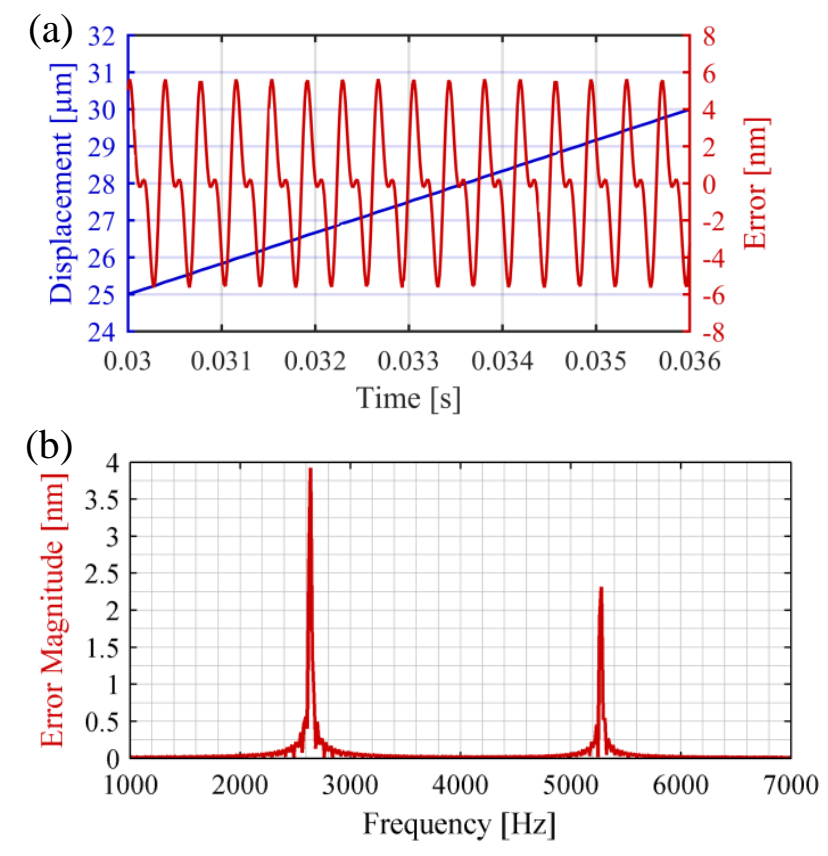

Fig. 4. (a) Simulated linear displacement at $50 \mathrm{~mm} / \mathrm{min}$ and periodic error with magnitudes of $4 \mathrm{~nm}$ and $2.5 \mathrm{~nm}$ for first and second order, respectively. (b) Periodic error amplitudes in the frequency domain.

Each order of the periodic error is modeled as a pure sine wave, $A \sin \theta(t)$, where $t$ is the time, $A$ is the amplitude, and $\theta$ is the phase. For example, first and second order periodic errors can be expressed as $A_{1} \sin \theta_{1}(t)+A_{2} \sin \theta_{2}(t)$. Fig. 5 shows first and second order periodic errors in both the time and spatial (polar coordinate) domains. The frequency, $f_{1}$, of the first order periodic error is half of the second order error frequency, $f_{2}$. Thus, the 
phase, $\theta_{1}$, of first order periodic error is half of the second order phase, $\theta_{2}$. In general, for $k^{\text {th }}$ order periodic error, the frequency, $f_{k}=k f_{1}$, and the phase $\theta_{k}=k \theta_{1}$.

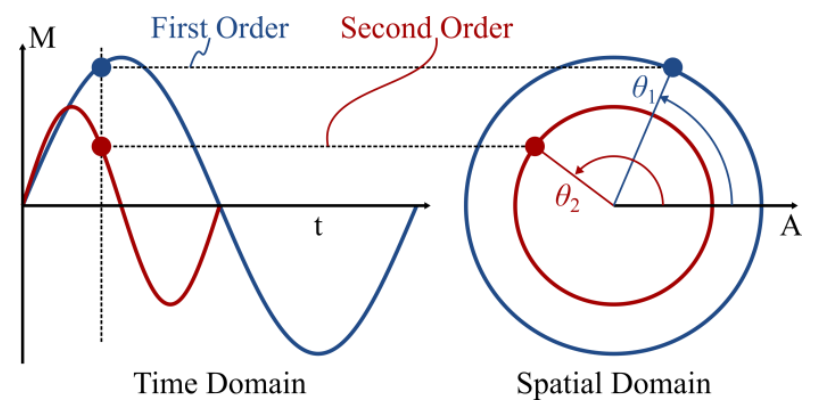

Fig. 5. First and second order periodic error in time and spatial domain.

Measured displacement data is collected at every sample interval, which means the data is discrete, such that the continuous form of CWT in Eq. (2.4) cannot be directly used in the algorithm. Instead, the discrete time continuous wavelet transform (DTCWT) is used

$$
W x(n, s)=\sum_{n^{\prime}=1}^{M}\left(x\left(n^{\prime}\right) \sqrt{s} \psi^{*}\left(\frac{\left(n^{\prime}-n\right) \Delta t}{s}\right) \Delta t\right),
$$

where $x(n)$ is the $n$th discrete data point, $\psi^{*}$ is the mother wavelet, $M$ is the number of total data points in the signal, and $\Delta t$ is the sampling time.

When the algorithm is implemented to post-process a measured displacement signal, the entire signal can be directly analyzed with the DTCWT since it is already known. However, when applying the algorithm in real-time (that is, a new displacement data point is received at each sampling time), only the present and previous data points are known. The DTCWT 
coefficient of one data point is calculated with its neighboring points. When calculating the DTCWT at the last point of the signal, half of the wavelet is outside the signal as shown in Fig. 6. Therefore, the DTCWT at the edges of the signal is not proportional to the DTCWT when the wavelet is almost entirely in the signal; this results in an "edge effect". There is no known method to eliminate this effect. However, a padding method (adding predicted points at the end of the signal) can partially resolve the issue. Zero-padding is commonly realized by adding zeroes at the end of the signal and it is used in the real-time DTCWTbased periodic error compensation algorithm proposed here. The real-time processing algorithm is described in the following paragraphs.

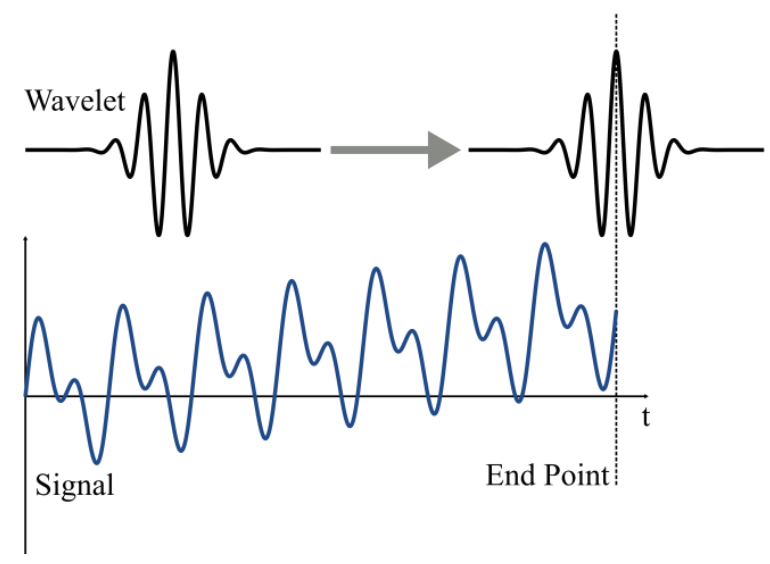

Fig. 6. The edge effect is depicted at the end of the signal.

The algorithm starts with storing the latest $N$ data points in a memory array, $X[1 \ldots N]$, which is used as the signal to conduct the DTCWT. First, detrending $X[1 \ldots N]$ is required to eliminate the main displacement component (subtracting the line connecting the beginning and ending points of the signal). This step is required because the magnitude of 
the periodic error is typically on the nanometer level while the overall displacement is usually on the micrometer level or larger. A new array $X^{\prime}[1 \ldots N]$ is obtained after detrending the measured data $X[1 \ldots N]$.

The DTCWT (Eq. 3.1) is applied to the signal $X^{\prime}[1 \ldots N]$ using the following five steps:

1) substitute the data points in $X^{\prime}[1 \ldots N]$ for $x$ in Eq. 3.1

2) select the mother wavelet to be the complex Morlet wavelet to produce child wavelets at various scales

3) set the shift parameter $\mathrm{n}$ to $\mathrm{N}$ (for the last point of the array)

4) build a scale array $s[1 \ldots M]$ to produce the child wavelets where $M$ is the total integer number of scales used in the DTCWT calculation

5) using Eq. 3.1 calculate the wavelet coefficient of the $N^{\text {th }}$ data point in the array

Because the complex Morlet wavelet has complex values the resulting coefficients from the DTCWT calculation in Eq. 3.1 will also be complex. Therefore, after applying the complex Morlet wavelet to the signal, the resulting wavelet transform is a complex array along the scale direction (see Fig. 7). 


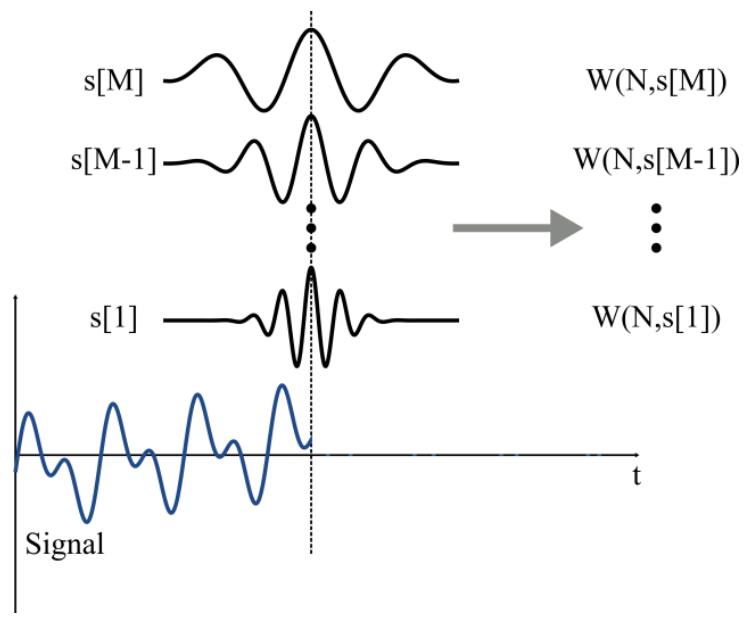

Fig. 7. DTCWT coefficients calculation at $n=N$ and scale $s[1 \ldots M]$.

The modulus and the phase for each complex coefficient can be calculated as:

$$
\begin{gathered}
a b s(n, s)=|W x(n, s)| \text { and } \\
\phi(n, s)=\arctan \left(\frac{\operatorname{Im}(W x(n, s))}{\operatorname{Re}(W x(n, s))}\right),
\end{gathered}
$$

where Im and Re represent the imaginary and real parts of the DTCWT coefficient, respectively. For the modulus $a b s(N, s)$ at $X^{\prime}[N]$ along the scale array, the maximum value of the DTCWT coefficient or "ridge" can be extracted. The ridge is defined as the location where the modulus reaches its local maximum at scale $s_{\text {ridge }}[46]$. When the modulus is maximal at the ridge, the frequency of the wavelet scaled by $s_{\text {ridge }}$ shows the greatest match with the convolved periodic error signal [47]. 
This $s_{\text {ridge }}$ equals $s_{1}$, which corresponds to the frequency of first order periodic error.

Therefore, the phase $\phi\left(N, s_{\text {ridge }}\right)$ is the first order periodic error phase at $X^{\prime}[N]$. A phase array $\varphi[1 \ldots N]$ is used to store this phase. A new point is added by completing two steps: 1$)$ remove $\varphi[1]$ and shift $\varphi[2 \ldots N]$ forward to $\varphi[1 \ldots N-1]$ and 2) set $\varphi[N]=\phi\left(N, s_{\text {ridge }}\right)$. Subsequently, the array $\varphi[1 \ldots N]$ has the first order periodic error phase information for the latest $N$ data points. Based on the periodic error model defined at the beginning of this section, with the phase array $\varphi[1 \ldots N]$ and an assumed unit amplitude, the $k^{\text {th }}$ order periodic error is $A_{k} \sin \left(\theta_{k}\right)=\sin (k \varphi)$. It is located at the scale $s_{k}=s_{1} / k$ since its frequency is $f_{k}=k f_{1}$ and the scale is inversely related to the frequency. The $k^{\text {th }}$ order periodic error for the latest $N$ points is

$$
r_{k}[1 \ldots N]=\{\sin (k \varphi(1)), \sin (k \varphi(2)), \ldots, \sin (k \varphi(N))\}
$$

which is called the "reference periodic error".

The next step is to determine the amplitude of different periodic error orders. The entire periodic error $e[1 \ldots N]$ is a linear combination of $m$ order periodic errors, which can be expressed as

$$
e[1 \ldots N]=\sum_{j=1}^{m} A_{j} r_{j}[1 \ldots N]
$$

where $A_{j}(j=1 \ldots m)$ is the periodic error amplitude on the $j^{\text {th }}$ order, which is to be quantified. 
Assuming that the detrended array, $X^{\prime}[1 \ldots N]$, is exactly the periodic error ${ }^{\dagger}$, the assumed sinusoidal combination of periodic errors $e[1 \ldots N]$ can be said to be equivalent to $X^{\prime}[1 \ldots N]$ according to Eq. 3.5 to obtain

$$
X^{\prime}[1 \ldots N]=\sum_{j=1}^{m} A_{j} r_{j}[1 \ldots N]
$$

The discrete form of the CWT in Eq. 3.1 can then be used on both sides of Eq. 3.6. Equation 3.6 is effectively substituting the actual periodic error for $x$ on one side of the equation and substituting the periodic error model on the other side of the equation. Once the values are substituted the complex Morlet wavelet can be used to calculate the coefficients by setting the location to be $n=N$, and using scales $s[1 \ldots M]$. The linearity property of the CWT introduced in Eq. 2.5 can then be used to construct the following result:

$$
W X^{\prime}[1 \ldots N](N, s[1 \ldots M])=\sum_{j=1}^{m} A_{j}\left[W r_{j}[1 \ldots N](N, s[1 \ldots M])\right]
$$

where $W X^{\prime}[1 \ldots N](N, s[1 \ldots M])$ has already been calculated. For $m$ order reference periodic errors, another $m$ DTCWT calculations about $W r_{j}[1 \ldots N](N, s) \quad(j=1 \ldots m)$ are required. Amplitudes $A_{j}(j=1 \ldots m)$ include $m$ unknowns, which require at least $m$ equations to be solved. Recall that the frequency of $j^{\text {th }}$ order periodic error is related to the scale $s_{j}=s_{1} / j$, so the DTCWT results $W X^{\prime}[1 \ldots N]\left(N, s_{i}\right)$ and $W r_{j}[1 \ldots N]\left(N, s_{i}\right)$ at scale

\footnotetext{
${ }^{\dagger}$ If there is a difference between the detrended signal and the actual periodic error due to imperfect detrending, this causes an error in the algorithm results.
} 
$s_{i}$ are extracted for use $(i, j=1 \ldots m)$. Let $c_{i}=W X^{\prime}[1 \ldots N]\left(N, s_{i}\right), d_{i j}=W r_{j}[1 \ldots N]\left(N, s_{i}\right)$, $i, j=1 \ldots m$. The following set of equations can then be obtained from Eq. 3.7:

$$
\left\{\begin{array}{c}
c_{1}=A_{1} d_{11}+A_{2} d_{12}+\ldots+A_{m} d_{1 m} \\
c_{2}=A_{1} d_{21}+A_{2} d_{22}+\ldots+A_{m} d_{2 m} \\
\vdots \\
c_{m}=A_{1} d_{m 1}+A_{2} d_{m 2}+\ldots+A_{m} d_{m m}
\end{array} \Rightarrow\left[\begin{array}{c}
A_{1} \\
A_{2} \\
\vdots \\
A_{m}
\end{array}\right]=\left[\begin{array}{cccc}
d_{11} & d_{12} & \cdots & d_{1 m} \\
d_{21} & d_{22} & \cdots & d_{2 m} \\
\vdots & \vdots & \ddots & \vdots \\
d_{m 1} & d_{m 2} & \cdots & d_{m m}
\end{array}\right]^{-1}\left[\begin{array}{c}
c_{1} \\
c_{2} \\
\vdots \\
c_{m}
\end{array}\right] .\right.
$$

The amplitudes $A_{j}(j=1 \ldots m)$ can be determined and then the magnitude $M$ of the periodic error at the latest sampling time is calculated using

$$
M=\sum_{i=1}^{m} A_{i} \sin (i \varphi(N))
$$

where $A_{i} \sin (i \varphi(N))$ is $\mathrm{i}^{\text {th }}$ order reconstructed periodic error at $n=N$. Finally, the magnitude $M$ is subtracted from the original displacement data to determine the compensated displacement data point.

Fig. 8 displays the sequence of calculations required for compensating one displacement data point in the DTCWT algorithm. 


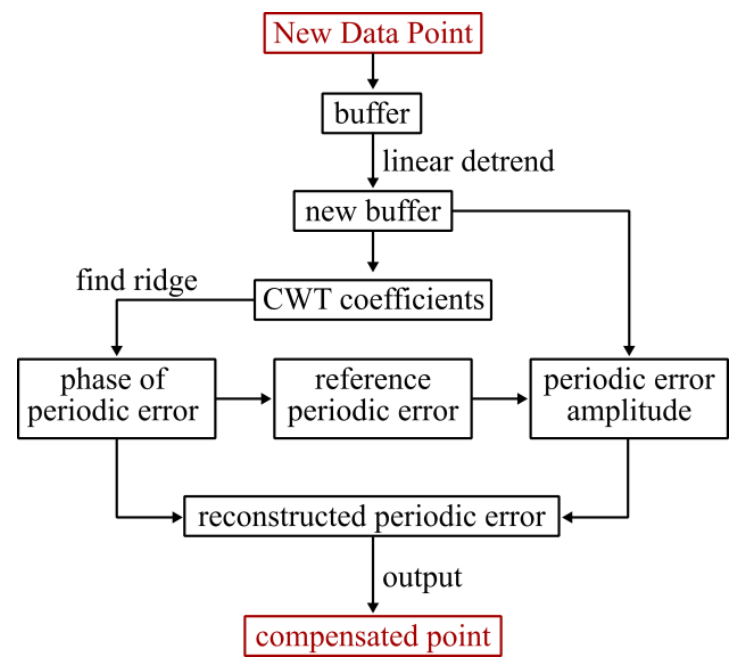

Fig. 8. Calculations to implement the periodic error compensation algorithm.

\section{Simulations and experiments}

Simulated and experimental displacement data with periodic errors were used to assess the validity of the wavelet-based compensation. Only the first and second order periodic errors were considered. The experimental parameters were: 1) He-Ne laser wavelength of $\lambda=$ $633 \mathrm{~nm} ; 2$ ) a fold factor of $F F=2$, which describes the number of light passes through the interferometer (the first order error completes a full cycle in $633 / 2=316.5 \mathrm{~nm}$, while the second order error requires $633 / 4=158.3 \mathrm{~nm}$ ); and 3) a sampling frequency was $62.5 \mathrm{kHz}$. The size $N$ of the array $X[1 \ldots N]$ was 200 in the simulations. In other words, at every sampling time, the latest 200 data points were used in the DTCWT compensation calculation. The choice of this size is based on the sampling rate and the frequency of periodic error, because the array needs to include several cycles of periodic error (at least 8 -10 cycles), in order to identify the error with high accuracy. If the sampling frequency is 
too high or the frequency of periodic error is too low, the array needs to be enlarged to accommodate enough periodic error cycles for calculation to achieve high accuracy in periodic error compensation.

The following sections discuss the results of: 1) constant velocity ridge detection, amplitude detection, and a comparison between the DTCWT and frequency domain approaches; and 2) non-constant velocity ridge detection, amplitude detection and periodic error compensation results.

\subsection{Ridge detection}

Identifying periodic error frequency components first requires ridge detection using Eq. 3.2. The performance of the ridge detection portion of the algorithm was evaluated using a simulated displacement signal where first and second order periodic errors (amplitudes 4 $\mathrm{nm}$ and $2.5 \mathrm{~nm}$, respectively) were superimposed during a constant velocity $(50 \mathrm{~mm} / \mathrm{min})$ displacement as shown in Fig. 4.

The real-time (point-by-point) algorithm shown in Fig. 8 was applied to this signal. The measured DTCWT ridge for this signal is displayed in Fig. 9 along with the result of the same algorithm implemented offline (no edge effects). The ridge detected from the realtime algorithm is at the integer scale $190 \pm 1$, while that from the off-line algorithm is consistently at scale 190 . This demonstrates that periodic uncertainty in the real-time 
algorithm caused by the edge effect can cause the calculated scale to differ from the actual value. However, the accuracy is still sufficient for real-time error compensation.

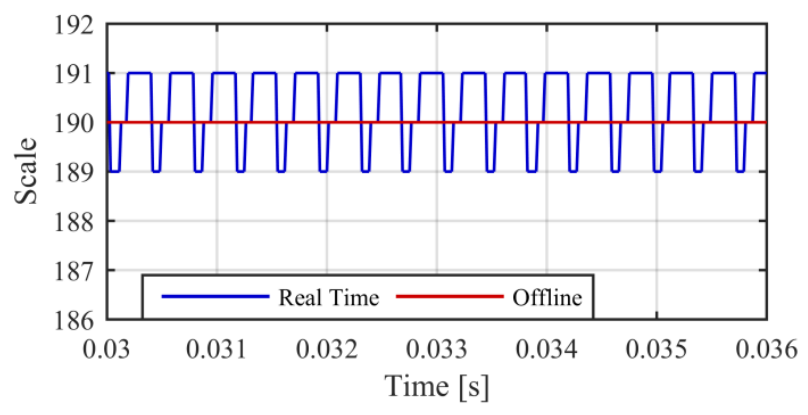

Fig. 9. The measured DTCWT ridge for the error signal.

\subsection{Amplitude measurement}

In the following tests, a simulated constant velocity motion $(50 \mathrm{~mm} / \mathrm{min})$ with a first order periodic error amplitude of $4 \mathrm{~nm}$ and second order periodic error amplitude of $2.5 \mathrm{~nm}$ was used just as in section 4.1. To identify the periodic error amplitudes under this constant velocity condition, two methods were compared at every sampling instant. The first method was a fast Fourier transform (FFT) method similar to [1-3]. The FFT of the error was computed after detrending the nominal displacement stored in the displacement array $X[1 \ldots N]$ and applying a Hanning window. The second method was the DTCWT-based algorithm. This algorithm was applied to calculate first and second order periodic error amplitudes (Eq. 3.8, where $m=2$ because only first and second order periodic errors exist) after obtaining the modulus and phase information (Eqs. 3.2 and 3.3) and determining the 
reference periodic errors (Eq. 3.4). The measured amplitudes are displayed in Fig. 10. The frequency domain approach result is smoother since windowing reduces the spectral leakage. The FFT assumes that the data is infinite and stationary. However, the signal used in the algorithm is finite (200 points, which is the same amount used in the DTCWT algorithm) and shifts forward by one data point each sampling interval. It actually measures the average amplitude over the signal. For first order periodic error, the true value of its amplitude is $4 \mathrm{~nm}$. The average value from the FFT approach is $3.92 \mathrm{~nm}$; the amplitude measured by the DTCWT approach is $4.25 \mathrm{~nm}$. For second order periodic error, the true value is $2.5 \mathrm{~nm}$. The amplitudes measured by the FFT and DTCWT approaches are $2.34 \mathrm{~nm}$ and $2.31 \mathrm{~nm}$, respectively. The two approaches show good agreement for amplitude measurement.

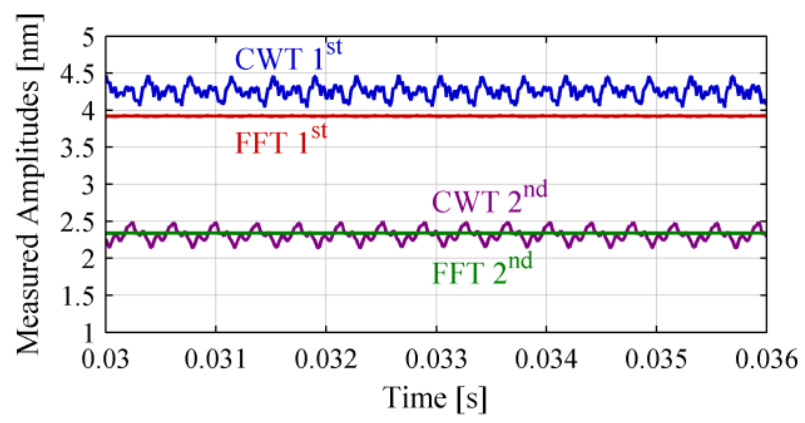

Fig. 10. The measured amplitudes for the FFT and DTCWT approaches.

\subsection{Constant velocity}


In these tests, the performance of the entire DTCWT algorithm (from receiving a new data point to providing a compensated data point) was examined. Again, the simulated 50 $\mathrm{mm} / \mathrm{min}$ constant velocity motion with superimposed periodic errors was used. The time domain periodic error compensation result is displayed in Fig. 11. The root-mean-square (RMS) error is reduced from $3.32 \mathrm{~nm}$ to $0.49 \mathrm{~nm}$ for both two methods. Fig. 12 displays the compensation result in the frequency domain. After compensation, the amplitudes of the first and second order periodic errors are reduced from $4 \mathrm{~nm}$ to $0.24 \mathrm{~nm}(0.27 \mathrm{~nm}$ for the FFT method) and from $2.5 \mathrm{~nm}$ to $0.30 \mathrm{~nm}(0.27 \mathrm{~nm}$ for the FFT method), respectively. These similar results indicate that the DTCWT algorithm has the capability to accurately compensate the periodic error.

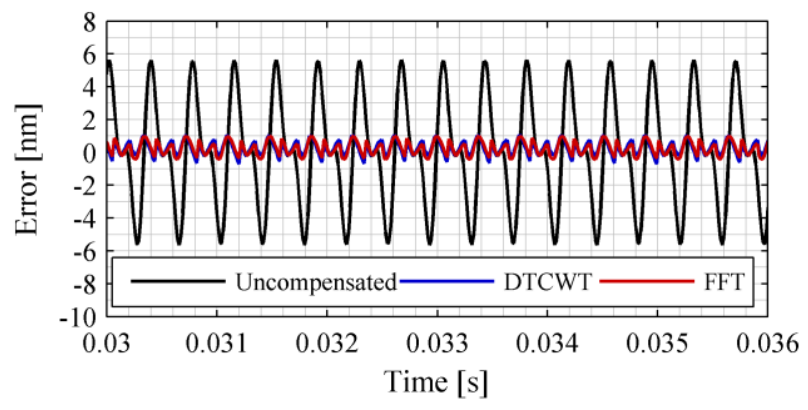

Fig. 11. The result of periodic error compensation (both DTCWT and FFT approaches) in the time domain is displayed. 

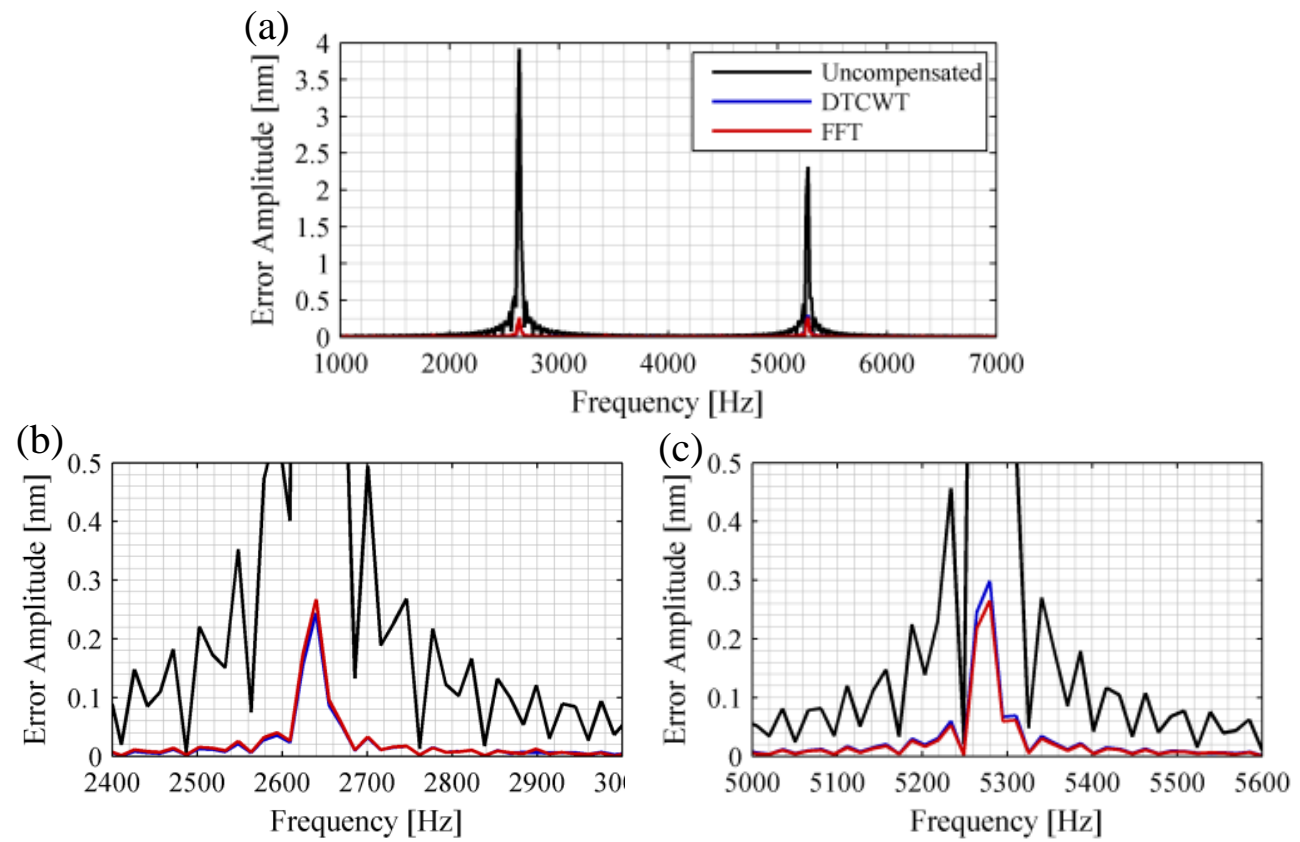

Fig. 12. (a) The result of periodic error compensation in the frequency domain is presented.

(b) Zoomed view of the compensation result for first order periodic error, and (c) for second order periodic error are demonstrated.

\subsection{Non-constant velocity}

One major advantage of the novel wavelet-based approach is that it can compensate periodic error during non-constant velocity motion. Not only did the wavelet-based approach demonstrate better results for constant velocity motions but the traditional frequency domain approach cannot be applied to non-constant motion profiles. This is because the FFT based approach to measuring periodic error amplitude assumes stationary signals during the period covered by the measurement array. However, for non-constant velocity motion, the periodic error time period and frequency is not constant although the 
spatial period is constant [42]. To demonstrate the effectiveness of the wavelet based approach, experimental data of a small stage was collected for a displacement of $300 \mu \mathrm{m}$ with parabolic velocity profile (due to constant acceleration of $50 \mathrm{~mm} / \mathrm{min}^{2}$ ). Fig. 13a shows the displacement and periodic error, which was isolated by subtracting a least squares fit polynomial from the displacement signal. The low frequency drift is caused by an imperfect polynomial fit or non-constant acceleration. The source of this low frequency content may also generically result from vibration or refractive index variation, for example. Fig. 13b also displays the compensated periodic error for 3um of motion. The DTCWT algorithm only removes the reconstructed periodic errors from the displacement. Other errors are not compensated and remain as residuals. These errors are implicitly included in the compensation calculation, as they cannot be eliminated with a low-order polynomial fit. This leads to spikes at some locations in the compensated result. Overall, the compensated result is results in significant periodic error compensation and reduces the RMS error by approximately $75.2 \%$. 

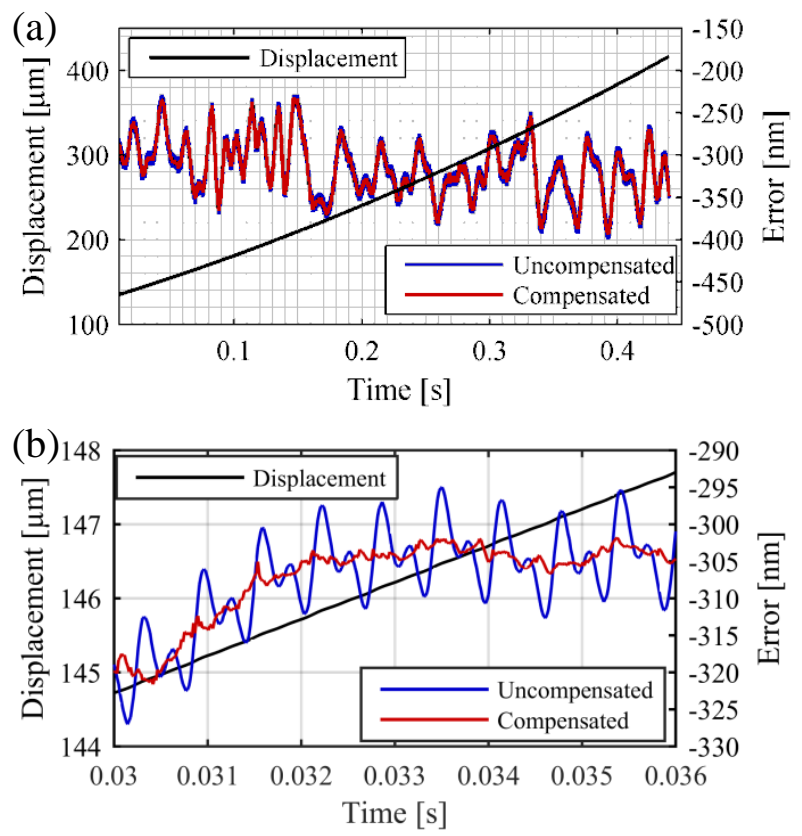

Fig. 13. (a) Experimental displacement for a $50 \mathrm{~mm} / \mathrm{min}^{2}$ constant acceleration and periodic error, and the result of periodic error compensation are demonstrated. (b) Zoomed view of the displacement and periodic error compensation result.

\section{Conclusions}

A new wavelet-based periodic error measurement and compensation method that can be used to compensate periodic errors for both constant and non-constant velocity profiles was presented. The performance of this approach was compared to the traditional frequency domain approach [1-3] under constant velocity conditions and demonstrated accurate compensation results. The algorithm was also used to compensate periodic error from nonconstant velocity motions. It was shown that it is possible to continuously identify periodic error under non-constant velocities and reconstruct the error in real-time reducing the RMS 
errors in experimental data by approximately $75 \%$. The algorithms presented in this work were designed to be executed on parallel hardware offering the potential application for real-time compensation of periodic error in heterodyne interferometers.

\section{Acknowledgement}

This work was partially supported by the National Science Foundation CMMI division under collaborative research awards 1265881, 1265842, and 1265824.

\section{References}

[1] Patterson S, Beckwith J. Reduction of systematic errors in heterodyne interferometric displacement measurement. In: Proceedings of the 8th International Precision Engineering Seminar (IPES). 1995. p. 101-4.

[2] Badami V, Patterson S. A frequency domain method for the measurement of nonlinearity in heterodyne interferometry. Precision Engineering 2000;24(1):41-9.

[3] Badami V, Patterson S. Investigation of nonlinearity in high-accuracy heterodyne laser interferometry. In: Proceedings of the 12th annual American Society for Precision Engineering (ASPE) conference. 1997. p. 153-6.

[4] Daubechies I. The wavelet transform, time-frequency localization and signal analysis. IEEE Transactions on Information Theory 1990;36(5):961-1005.

[5] Lu C, Gillmer SR, Ellis JD, Schmitz TL, Troutman JR, Tarbutton JA. Application of wavelet analysis in heterodyne interferometry. In: Proceedings of the 28th annual American Society for Precision Engineering (ASPE) conference. 2013. p. 364-7. 
[6] Lu C, Troutman JR, Ellis JD, Schmitz TL, Tarbutton JA. Periodic error compensation using frequency measurement with continuous wavelet transform. In: Proceedings of the 29th annual American Society for Precision Engineering (ASPE) conference. 2014. p. 40811.

[7] Lu C, Ellis JD, Schmitz TL, Tarbutton JA. Improvement of a periodic error compensation algorithm based on the continuous wavelet transform. In: Proceedings of the 30th annual American Society for Precision Engineering (ASPE) conference. 2015.

[8] Fedotova G. Analysis of the measurement error of the parameters of mechanical vibrations. Measurement Techniques 1980;23(7):577-80.

[9] Quenelle R. Nonlinearity in interferometric measurements. Hewlett-Packard Journal 1983;34(4):10.

[10] Sutton C. Nonlinearity in length measurements using heterodyne laser Michelson interferometry. Journal of Physics E: Scientific Instrumentation 1987;20:1290-2.

[11] Barash VY, Fedotova G. Heterodyne interferometer to measure vibration parameters. Measurement Techniques 1984;27(1):50-1.

[12] Bobroff N. Residual errors in laser interferometry from air turbulence and nonlinearity. Applied Optics 1987;26(13):2676-82.

[13] Rosenbluth A, Bobroff N. Optical sources of non-linearity in heterodyne interferometers. Precision Engineering 1990;12(1):7-11.

[14] Stone JA, Howard LP. A simple technique for observing periodic nonlinearities in Michelson interferometers. Precision Engineering 1998;22(4):220-32.

[15] Cosijns S, Haitjema H, Schellekens P. Modeling and verifying non-linearities in heterodyne displacement interferometry. Precision Engineering 2002;26(4):448-55. 
[16] Wu C-M, Deslattes RD. Analytical modeling of the periodic nonlinearity in heterodyne interferometry. Applied Optics 1998;37(28):6696-700.

[17] Wu C-m, Su C-s. Nonlinearity in measurements of length by optical interferometry. Measurement Science and Technology 1996;7(1):62.

[18] Hou W, Wilkening G. Investigation and compensation of the non-linearity of heterodyne interferometers. Precision Engineering 1992;14(2):91-8.

[19] Hou W, Zhao X. Drift of nonlinearity in the heterodyne interferometer. Precision Engineering 1994;16(1):25-35.

[20] Howard L, Stone J. Computer modeling of heterodyne interferometer errors. Precision Engineering 1995;12(1):143-6.

[21] Tanaka M, Yamagami T, Nakayama K. Linear interpolation of periodic error in a heterodyne laser interferometer at subnanometer levels [dimension measurement]. Ieee Transactions on Instrumentation and Measurement 1989;38(2):552-4.

[22] Wu C-m, Lawall J, Deslattes RD. Heterodyne interferometer with subatomic periodic nonlinearity. Applied Optics 1999;38(19):4089-94.

[23] Schmitz T, Evans C, Davies A, Estler WT. Displacement uncertainty in interferometric radius measurements. CIRP Annals-Manufacturing Technology 2002;51(1):451-4.

[24] Joo K-N, Ellis JD, Spronck JW, van Kan PJ, Schmidt RHM. Simple heterodyne laser interferometer with subnanometer periodic errors. Optics Letters 2009;34(3):386-8.

[25] Schmitz T, Kim HS. Monte Carlo evaluation of periodic error uncertainty. Precision Engineering 2007;31(3):251-9.

[26] Bobroff N. Recent advances in displacement measuring interferometry. Measurement Science and Technology 1993;4(9):907. 
[27] Steinmetz C. Sub-micron position measurement and control on precision machine tools with laser interferometry. Precision Engineering 1990;12(1):12-24.

[28] Cretin B, Xie W-X, Wang S, Hauden D. Heterodyne interferometers: practical limitations and improvements. Optics Communications 1988;65(3):157-62.

[29] Petrů F, Číp O. Problems regarding linearity of data of a laser interferometer with a single-frequency laser. Precision Engineering 1999;23(1):39-50.

[30] Augustyn W, Davis P. An analysis of polarization mixing errors in distance measuring interferometers. Journal of Vacuum Science \& Technology B 1990;8(6):2032-6.

[31] Xie Y, Wu Y-z. Zeeman laser interferometer errors for high-precision measurements. Applied Optics 1992;31(7):881-4.

[32] De Freitas J, Player M. Importance of rotational beam alignment in the generation of second harmonic errors in laser heterodyne interferometry. Measurement Science and Technology 1993;4(10):1173.

[33] De Freitas J, Player M. Polarization effects in heterodyne interferometry. Journal of Modern Optics 1995;42(9):1875-99.

[34] De Freitas JM. Analysis of laser source birefringence and dichroism on nonlinearity in heterodyne interferometry. Measurement Science and Technology 1997;8(11):1356.

[35] Li B, Liang J-w. Effects of polarization mixing on the dual-wavelength heterodyne interferometer. Applied Optics 1997;36(16):3668-72.

[36] Park B, Eom T, Chung M. Polarization properties of cube-corner retroreflectors and their effects on signal strength and nonlinearity in heterodyne interferometers. Applied Optics 1996;35(22):4372-80. 
[37] Oldham N, Kramar J, Hetrick P, Teague E. Electronic limitations in phase meters for heterodyne interferometry. Precision Engineering 1993;15(3):173-9.

[38] Schmitz T, Beckwith JF. An investigation of two unexplored periodic error sources in differential-path interferometry. Precision Engineering 2003;27(3):311-22.

[39] Eom T, Choi T, Lee K, Choi H, Lee S. A simple method for the compensation of the nonlinearity in the heterodyne interferometer. Measurement Science and Technology 2002;13(2):222-5.

[40] Eom TB, Kim JA, Kang C-S, Park BC, Kim JW. A simple phase-encoding electronics for reducing the nonlinearity error of a heterodyne interferometer. Measurement Science and Technology 2008;19(7):075302 (6pp.).

[41] Schmitz T, Chu DC, Kim HS. First and second order periodic error measurement for non-constant velocity motions. Precision Engineering 2009;33(4):353-61.

[42] Schmitz T, Adhia C, Kim HS. Periodic error quantification for non-constant velocity motion. Precision Engineering 2012;36(1):153-7.

[43] Ellis JD, Baas M, Joo K-N, Spronck JW. Theoretical analysis of errors in correction algorithms for periodic nonlinearity in displacement measuring interferometers. Precision Engineering 2012;36(2):261-9.

[44] Farge M. Wavelet transforms and their applications to turbulence. Annual Review of Fluid Mechanics 1992;24(1):395-458.

[45] Dursun A, Özder S, Ecevit FN. Continuous wavelet transform analysis of projected fringe patterns. Measurement Science and Technology 2004;15(9):1768.

[46] Liu H, Cartwright AN, Basaran C. Moire interferogram phase extraction: a ridge detection algorithm for continuous wavelet transforms. Applied Optics 2004;43(4):850-7. 
[47] Cherbuliez M, Jacquot P. Phase computation through wavelet analysis: yesterday and nowadays. Fringe. 2001. p. 154-62. 


\section{Figure captions}

Fig. 1. Schematic of heterodyne interferometer setup. Optical components include:

retroreflectors $(\mathrm{RR})$, polarizing beam splitter $(\mathrm{PBS})$, polarizers, half wave plate (HWP), and photodetectors.

Fig. 2. Translation and dilation of the mother wavelet. (a) Shifting a wavelet in time is translation. (b) Stretching or squeezing a wavelet is dilation.

Fig. 3. The mother wavelet of the complex Morlet wavelet.

Fig. 4. (a) Simulated linear displacement at $50 \mathrm{~mm} / \mathrm{min}$ and periodic error with magnitudes of $4 \mathrm{~nm}$ and $2.5 \mathrm{~nm}$ for first and second order, respectively. (b) Periodic error amplitudes in the frequency domain.

Fig. 5. First and second order periodic error in time and spatial domain.

Fig. 6. The edge effect is depicted at the end of the signal.

Fig. 7. DTCWT coefficients calculation at $n=N$ and scale $s[1 \ldots M]$.

Fig. 8. Calculations to implement the periodic error compensation algorithm.

Fig. 9. The measured CWT ridge for the error signal.

Fig. 10. The measured amplitudes for the FFT and DTCWT approaches.

Fig. 11. The result of periodic error compensation (both DTCWT and FFT approaches) in the time domain is displayed.

Fig. 12. (a) The result of periodic error compensation in the frequency domain is presented.

(b) Zoomed view of the compensation result for first order periodic error, and (c) for second order periodic error are demonstrated. 
Fig. 13. (a) Experimental displacement for a $50 \mathrm{~mm} / \mathrm{min}^{2}$ constant acceleration and periodic error, and the result of periodic error compensation are demonstrated. (b) Zoomed view of the displacement and periodic error compensation result. 